\title{
Polyhistidine Affinity Chromatography: From the Idea to a Method of Broad Applicability and the Pitfalls in-between
}

\author{
Heinz Döbeli*
}

\begin{abstract}
The present article recapitulates the development of the polyhistidine affinity tag purification principle. Emphasis is laid on events behind the scenes which were never published. The key concept of the method emerged in a team discussion and its further development was driven by the need of Roche in-house projects.
\end{abstract}

Keywords: Affinity tag removal $\cdot$ Histidine affinity tag $\cdot$ Nickel chelate $\cdot$ Ni-NTA $\cdot$ Protein folding $\cdot$ Protein purification

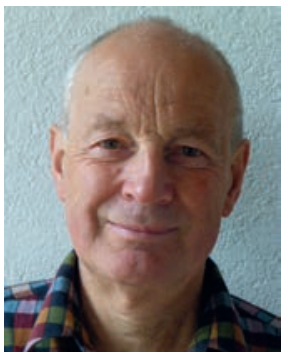

Heinz Döbeli was born on the $13^{\text {th }}$ March 1951 in Liestal, where he attended the local school system until the Matura in 1970. He studied chemistry at the University Basel, graduating in 1974. About half of the class including Oreste Ghisalba were headhunted by the newly established Biocenter to follow their PhD programs. Oreste and Heinz found positions in Ciba-Geigy AG where their microbiology professor, Dr. Jakob Nüesch, was head of the microbiology department. The four years with Oreste had a profound impact on Heinz's view on the world. Science was a topic, but politics were hotter. After his PhD in Biochemistry in 1979 Heinz took a post doc position at the University Hospital in Basel and 1984 he joined F. Hoffmann-La Roche AG, where he stayed until his retirement in 2013. Heinz's expertise was in protein chemistry and because most project groups required this expertise, he worked in very diverse indications: Interferon production, AIDS, malaria, Alzheimer and metabolic diseases.

\section{Memories of a Creative Meeting}

It was not intended to make an invention in a protein downstreaming lab heads' meeting, on a normal Wednesday morning in spring 1985 at Hoffmann-La Roche, Basel. The invention which finally gained world-wide recognition comprises a method that allows the easy purification of recombinant proteins. The ingredients for that eureka experience were three running projects and a few words from a colleague who reported from a scientific meeting.

At these weekly meetings, progress and problems of protein purification activities were discussed. At that time, three recombinant proteins produced in $E$. coli were in development: $\alpha$-interferon, $\beta$-interferon and $\gamma$-interferon. One step in the purification protocol of $\alpha$-interferon comprised a copper chelate chromatography column which had been designed by our group leader, Erich Hochuli. This step was critical to remove a side product generated in the fermentation process. Copper has four coordination sites. Three were exploited to immobilize copper to the chromatography gel via an iminodiacetic acid (IDA) moiety. ${ }^{[1]}$ The fourth coordination site was used to bind the protein via an amino group, a histidine residue or a sulfur group.
Bernhard Eggimann showed that this column type may also be useful for the purification of $\beta$-interferon. Two histidine residues positioned in an $\alpha$-helix were forced into a proximal position. In order to exploit these fortuitously positioned histidines for affinity purification the immobilized metal has to provide two coordination sites. Therefore, copper was replaced by nickel. Nickel has six coordination sites. Three are required to immobilize the metal, two for binding the histidines and one is redundant (Fig. 1). With this early design of our metal affinity columns, leakage of nickel into the purified protein was unfortunately observed. Heavy metal ions in a preparation supposed to become a therapeutic protein is a no-go.

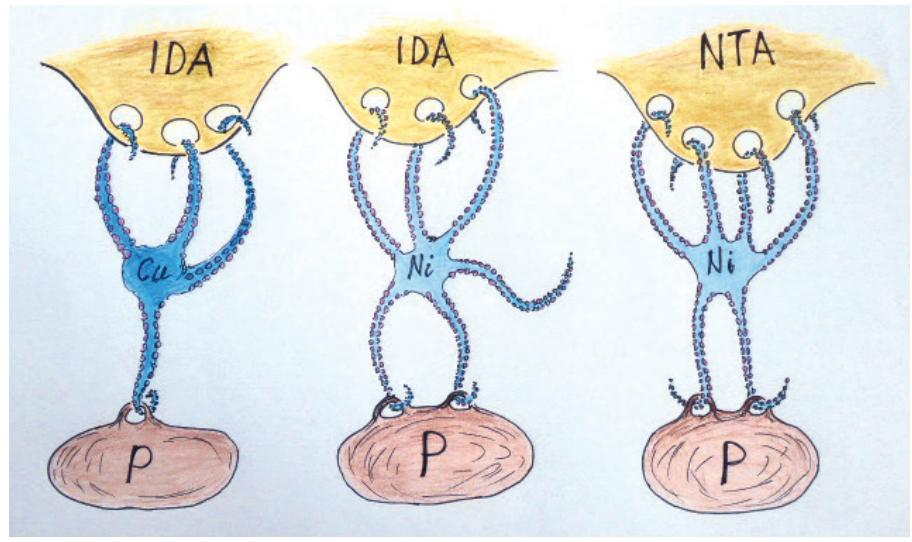

Fig. 1. Coordination number and ligands matter. Copper can bind ligands through four coordination sites, nickel through six. Iminodiacetic acid (IDA) is a tridentate ligand, nitrilotriacetic acid (NTA) a tetradentate ligand. When IDA is immobilized to a chromatography gel loaded with $\mathrm{Cu}^{2+}$, one coordination site is free and can bind to a histidine, an amino group or to a sulfhydryl group of a protein. Although this binding is strong, it lacks specificity. When $\mathrm{Cu}^{2+}$ is replaced by $\mathrm{Ni}^{2+}$ two coordination sites may bind to suitably positioned groups of a protein and thus lead to specificity. However, when a protein contains three proximal handles the binding might be so strong that $\mathrm{Ni}^{2+}$ remains bound to the protein and appears in the eluate (see example with the peptide HHLGGAKQAGDV). NTA offers a predetermined breaking point between the protein and the Ni-NTA complex (exception: carboxy-terminal HHHHHC). The colors of the $\mathrm{Cu}$-tetrabus and $\mathrm{Ni}$-hexabus correspond to the real colors of the chromatography gels (tetrabus and hexabus are octopus-like animals devoid of four or two tentacles). We also considered other metals able to act as an octobus, but when we realized that Ni-NTA fulfilled all our needs, we stopped any investment. P stands for protein and the handles represent histidines. Cartoon drawn by the author. 
I was responsible for $\gamma$-interferon purification. Unlike $\alpha$ - and $\beta$-interferon, $\gamma$-interferon does not contain histidine clusters that might coordinate with immobilized metal ions. However, it does contain seven basic amino acids at its carboxy-terminus that allow efficient purification with a cation exchanger. This reminded Bernhard of a recently attended meeting. Smith and coworkers ${ }^{[2]}$ reported the purification of a recombinant protein, artificially enlarged at its carboxy-terminus by several arginine residues, that allowed easy purification. Removal of the artificial arginine residues was performed with carboxy peptidase B to yield the desired protein. We resolved to build on the work of Smith et al. for $\gamma$-interferon, but use histidine instead of arginine and place the recombinant amino acids at the N-terminus. This would enable metal affinity chromatography, while a proteolytic cleavage site immediately following the artificial residues would expose the important glutamine and enable its conversion to pyroglutamic acid (Table 1). Thus, proteolytic cleavage, to expose the important glutamine, was the main driver of the almost incidental His-tag invention.

Purification was not the problem but instead a methionine residue at the amino-terminus. The first codon on the transfer RNA is AUG which codes for a methionine. Therefore, recombinant proteins expressed in E. coli all start with a methionine. Depending on the following amino acid residue this initiator methionine is cleaved off or not. In our expression plasmid the second amino acid is glutamine and this prevents removal.

In humans, $\gamma$-interferon is a secretory protein and is therefore produced differently. The $\gamma$-interferon gene contains the initiator methionine followed by a signal sequence. This stretch of hydrophobic amino acids is responsible for the export into the endoplasmic reticulum. A signal peptidase then cleaves off this signal sequence to generate a $\gamma$-interferon version which starts with a glutamine residue which is later cyclized into a pyroglutamic acid residue.

In order to obtain a $\gamma$-interferon version also starting with a glutamine residue (which can be cyclized chemically) we generated an expression plasmid starting with a methionine, followed by the histidine affinity tag, a protease cleavage site and the $\gamma$-interferon sequence where the first amino acid is glutamine.

These concepts together with metal chelate chromatography and neighboring histidine residues was our eureka. We decided to ask our friends from the Gene Technology unit, Rainer Gentz and Dietrich Stüber, to construct an expression plasmid of $\gamma$-interferon equipped with two neighboring histidines and a proteolytic cleavage site between the native interferon sequence and the histidine affinity tag (Table 1).

\section{From the Idea to a Prototype}

At that time, synthesizing a novel piece of DNA was a tedious work. Willy Bannwarth spent several months to construct such a piece of DNA. In order to use the waiting time, I started to perform experiments with model peptides and proteins.

The first experiment was a complete failure, which nevertheless provided interesting information. I applied a small peptide with the sequence HHLGGAKQAGDV to an IDA nickel chelate column hoping it would be bound on the matrix. However, the upper part of the light blue chromatography gel became white as the nickel was leached from the matrix and the peptide passed directly through the column taking the nickel with it - very similar to the experience with $\beta$-interferon. We realized that an IDA column (a half-EDTA) is not suitable to retain nickel when a peptide with two neighboring histidines plus a free $\mathrm{N}$-terminal amino group is chromatographed.

Erich succeeded in synthesizing a nitrilotriacetic acid (NTA) derivative (Fig. 1) which was able to bind the above-mentioned peptide, in combination with nickel, as well as many more peptides and proteins. ${ }^{[3,4]}$ An elegant proof of principle were muscle-type and heart-type lactic dehydrogenases. The muscle-type isoenzyme contains the sequence motif $\mathbf{H V P H}$ which results in binding to an Ni-NTA column. The heart-type isoenzyme does not contain such a sequence element and is not retained despite 28 single isolated histidine residues.

\section{First Recombinant His-tagged Protein}

Finally, our colleagues succeeded in constructing a DNA sequence encoding a histidine tagged $\gamma$-interferon with the aminoterminal sequence MHHAGIEGRQDP (Table 1).

The four amino acids IEGR represent the cleavage recognition site of the blood coagulation factor Xa. The chromatographic behavior on a Ni-NTA column of that $\gamma$-interferon version fully satisfied our expectations. However, we observed a decreased antiviral activity compared with the sequence of the parent version MQDP (Table 1). We assumed that this extra piece of sequence inhibits the activity. In humans $\gamma$-interferon is expressed with a signal peptide which is cleaved off by a signal peptidase. Our speculation was that a $\gamma$-interferon version starting with a glutamine or even better with its cyclisation product pyroglutamate should have a higher anti-viral activity.

Treatment with factor Xa generated a $\gamma$-interferon with a slightly lower molecular weight as shown by SDS gel electrophoresis. In addition, the anti-viral activity was increased. Our hopes were raised, but not for long. Amino-terminal sequencing revealed a sequence still beginning with MHHAGIEGRQDP. By analyzing

Table 1. Sequence and anti-viral activity of recombinant $\gamma$-interferon versions

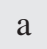

b

c

d

e amino-terminus

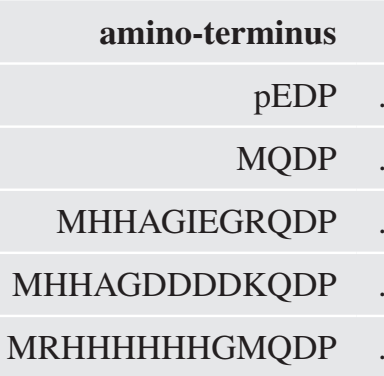

\section{carboxy-terminus}

GKRKRSQMLFRGRRASQ

GKRKRSQMLFRGRRASQ

GKRKRSQMLFRGRRASQ

GKRKRSQMLFRGRRASQ

GKRKRSQMLFRGRRASQ

\begin{tabular}{l}
$?$ \\
$100 \%$ \\
$30 \%$ \\
\hline $5 \%$ \\
\hline $5 \%$
\end{tabular}

a: Mature human $\gamma$-interferon with a pyroglutamate $(\mathrm{pE})$ residue at the amino-terminus, b: Recombinant $\gamma$-interferon from $E$. coli still bearing the initiator methionine, c: His-tagged version with a factor Xa cleavage site (IEGR), d: version with an enterokinase cleavage site (DDDDK) and a His-tagged version with six consecutive histidines.

Amino acids are given as their one-letter codes. $\mathrm{pE}$ stands for pyroglutamate. This residue is generated by cyclisation of an aminoterminal glutamine residue. We never succeeded to generate an $E$. coli recombinant version containing a pyroglutamate residue as is the case for $\gamma$-interferon generated in humans. 
the literature in more detail, we realized that factor Xa does not cleave when the first or second amino acid after the cleavage site carries a negative charge. It took quite an experimental effort to prove that factor Xa cleavage occurred at the carboxy-terminus. Also, we found out that deletion of few carboxy-terminal amino acids increase the anti-viral activity. ${ }^{[5]}$

As we will see later, removal of artificial tags from recombinant proteins continued to be tricky. There is a big gap between the specificity of proteases reported in textbooks and the specificity experienced in real experiments.

\section{Next Challenge: Purification of Insoluble Protein Constructs}

This section explains why most researchers worldwide use affinity tags with six consecutive histidines and not two, three, four, five or seven.

During the eighties AIDS was a hot topic in pharmaceutical companies. The ability to sequence the genome of an entire virus and the possibility to produce recombinant proteins raised hope to use this technology to generate diagnostic screening assays or even vaccines.

The concept of our colleagues from Gene Technology was to select three immuno-dominant sequences each from a different protein and combine them in one artificial protein. This protein did not yet contain a histidine affinity tail. However, this artificial fusion protein and many more constructs tested were insoluble in all buffer systems generally used for protein purification. Only sodium dodecyl sulfate (SDS) containing buffers, $8 \mathrm{M}$ urea or $6 \mathrm{M}$ guanidinium hydrochloride $(\mathrm{Gu}-\mathrm{HCl})$ were able to dissolve these proteins. To generate a screening assay for HIV the proteins must be highly pure. E. coli contaminations may lead to an intolerable rate of false positive results. The most effective methods like antibody columns did not withstand these non-physiological conditions (Fig. 2).

By investigating histidine-containing model peptides or proteins, I realized that small peptides were retained even in the presence of high concentrations of $\mathrm{Gu}-\mathrm{HCl}$. Proteins with similar histidine configurations were also retained, albeit at a lower Gu$\mathrm{HCl}$ concentration. Whether proteins or peptides are retained appeared to be a function of the histidine arrangement and the size of the protein. Based on this knowledge Rainer and Dietrich started to systematically increase the number of histidines either at the amino- or at the carboxy-tail of the model protein dihydrofolate reductase. ${ }^{[6,7]}$

Affinity tails with six consecutive histidines, either aminoor carboxy-terminal became a Roche internal standard and after our publication, also outside of Roche. With this type of label almost all buffer systems including $6 \mathrm{M} \mathrm{Gu}-\mathrm{HCl}$ were applicable unless they were free of chelating compounds such as EDTA or dithiolcompounds (e.g. dithiothreitol). SDS is also very rarely tolerated.

The first commercial protein with an artificial histidine affinity tag was the above-mentioned artificial HIV construct. A gene sequence coding for an affinity-tag with six consecutive histidines was fused amino-terminal to the gene coding for the HIV protein construct. This highly artificial protein was expressed in E. coli and extracted in $6 \mathrm{M} \mathrm{Gu}-\mathrm{HCl}$. After a nickel-NTA column in 6 $\mathrm{M} \mathrm{Gu}-\mathrm{HCl}$ and a size-exclusion column in an SDS containing buffer the protein was pure enough to be integrated into an AIDS diagnostic test-kit.

There was a grim competition among providers of HIV tests. When a new serotype of the virus was detected, a race for an update of the test started. Thanks to our standardized purification method we were quite quick in generating new protein versions.

With tags containing six histidines the Ni-NTA chromatography was applicable in almost all buffer systems, the 6-His tag became many protein chemist's only tag.
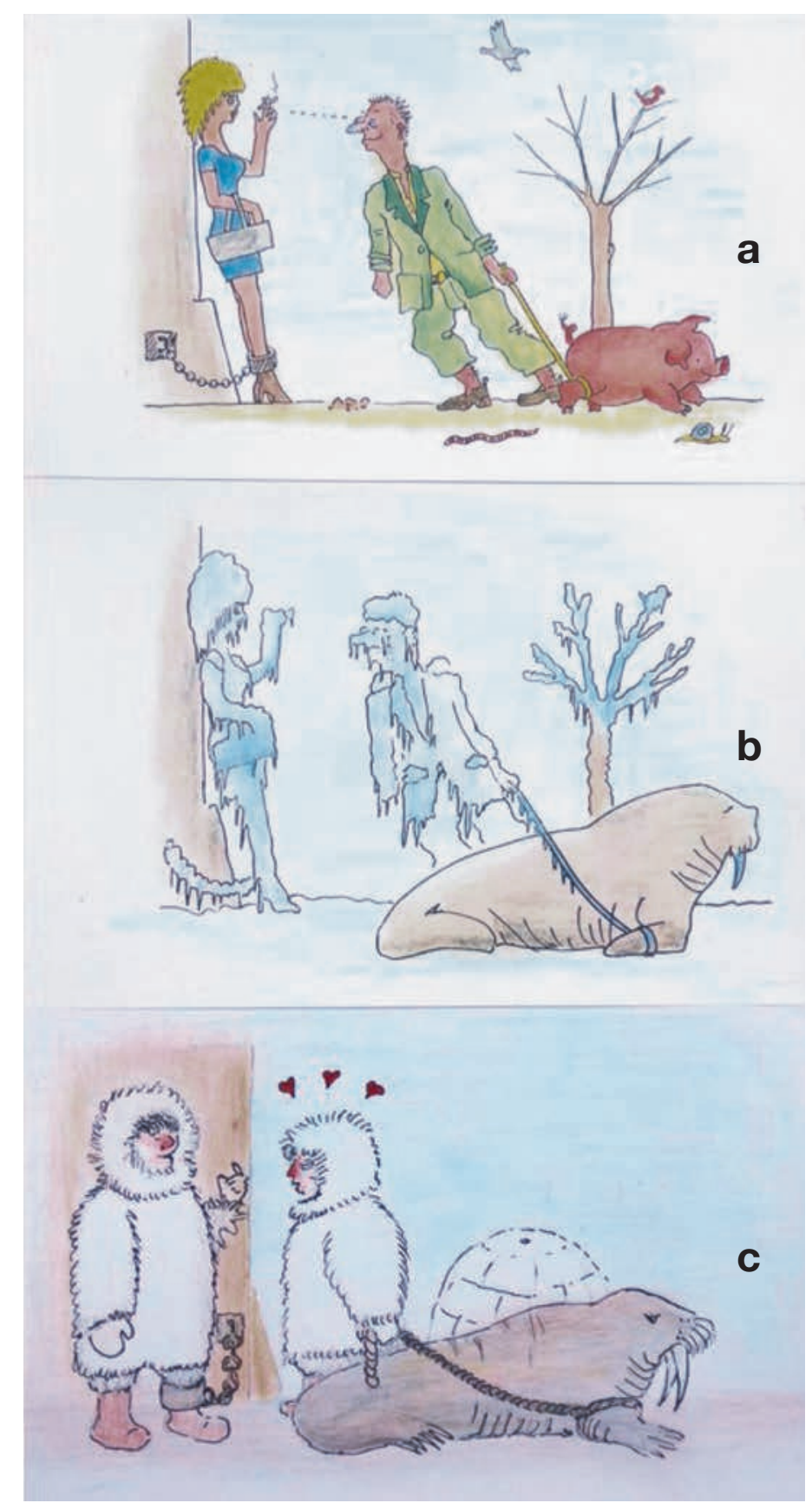

Fig. 2. Physiological conditions and beyond. Affinity purification based on biological principles such as antibodies is very efficient as symbolized by cartoon (a). Cartoon (b) stands for unfriendly conditions which have to be applied to make the walrus happy. But the unfriendly conditions prevent any biological affinity. In cartoon (c) the affinity system is adapted to the favorite conditions of the walrus. The break through of Ni-NTA method arose thanks to its broad applicability even under strong denaturing conditions. Cartoon drawn by Dr. Edi Riesen, Ziefen.

\section{Carboxy-terminal His-tags: Optimal for Membrane Proteins}

Membrane proteins represent a challenge for protein purification because of their very hydrophobic nature. When only the extracellular part of the protein was of interest, we - and later many others - replaced the membrane anchor by a histidine affinity tag. My first experiment was with the surface glycoprotein of HIV (gp120). Because of the heavy glycosylation, this protein was not retained either by anion or cation exchangers and not by hydrophobic interaction columns. The methods of choice became nickel chelate and size exclusion chromatography, which led to a pure protein. 
Since the extracellular part of such proteins are mostly glycosylated, we standardized the procedure by applying first a concanavalin A column followed by a nickel chelate column. The concanavalin A step was useful to remove many components of the fermentation broth and to transfer the desired protein into a defined buffer system suitable for the Ni-NTA chromatography.

Hugues Matile, an immunologist, succeeded very soon to generate anti-His-tag antibodies which turned out to be useful in detecting the tagged proteins on western blots or in labeling of cells treated with tagged proteins.

\section{Removal of the His-tag from a Self-aggregating Peptide}

Some colleagues named it the Devil's peptide because of its nasty properties. In the literature it is called amyloid peptide and is detected in the amyloid plaques of Alzheimer's disease patients. According to the amyloid hypothesis a larger membrane protein is cleaved, first in the extracellular part and then inside its transmembrane anchor to liberate a peptide with a variable length of 39 to 42 amino acid residues. Synthetic versions of these peptides, in particular the 42-residue peptide, tend to aggregate into fibers, which are virtually identical to those found in the plaques of Alzheimer's disease patients.

In the nineties, most scientists involved in Alzheimer's research were firmly convinced that generation and aggregation of these peptides intoxicate neurons and thus are the cause of the disease. Many projects were based on approaches to prevent generation of these peptides or to ameliorate its neurotoxicity by an antidote. For these studies a vast quantity of peptides and many variants thereof were required. Synthesis of large peptides is tricky, in particular when long stretches of hydrophobic amino acids are part of the sequence. Also, incomplete coupling during synthesis leads to side products which are difficult to remove, and chemical synthesis is expensive. In 1990 we got a quote from a company specialized in peptide synthesis: $25^{\prime} 000$ Swiss francs for $25 \mathrm{mg}$ of the 42-residue peptide. Therefore, Roche scientists opted for a biotechnical production. By the end of the project, we had produced dozens of grams of recombinant amyloid peptide for our research programs.

We constructed fusion proteins containing a histidine affinity tail, a bulky hydrophilic repeat unit of 19 NANP residues and a cleavage site followed by the human amyloid peptide. The NANP repeat originated from a Plasmodium falciparum protein. It was found that proteins containing this sequence result in a high expression yield in E. coli. Only later we realized that the effective advantage is the fact that this highly water-soluble bulky part prevents the aggregation of the protein construct. We never succeeded in obtaining amyloid peptides in the desired quality when we constructed shorter histidine tags devoid of this NANP repeat.

Since the amyloid peptide contains sequence elements which were expected to be split by most of the proteases suitable to remove the tag, we introduced amino acids designed to be cleaved by chemical methods: Asp-Pro for limited acid cleavage, Trp for BNPS-scatole, Asn-Gly for hydroxylamine and Cys for 2-nitro5-thiocyanatobenzoic acid cleavage. None of these textbook-derived methods led to convincing results.

As a back-up, a methionine-inserted version for cyanogen bromide $(\mathrm{CNBr})$ cleavage was constructed despite the presence of an internal methionine at the carboxy-terminus of the amyloid peptide. We speculated to be able to get some full-length peptide by limited cleavage. This was accomplished by adsorbing the fusion protein on a reversed phase C18-HPLC column. We assumed that the hydrophilic NANP repeat moiety remains in the aqueous phase whereas the methionine surrounded by hydrophobic residues in the membrane anchor part is buried in the C18-hydrophobic matrix. We repurposed an HPLC column as a chemical reactor. This method was successful. It led to a preparation containing up to $85 \%$ amyloid peptide. The rest was noncleaved fusion protein still containing the histidine tail and the NANP-repeat sequence. ${ }^{[8]}$

\section{Transfer of the Amyloid Peptide into a Physiological Buffer}

Another obstacle was aggregation of the peptide as soon as it was split off from the bulky fusion tail and transferred into a physiological buffer system. A peptide which aggregates during its production is a nightmare for protein chemists.

The entire procedure comprised four columns: a first $\mathrm{Ni}-$ NTA column to purify the fusion protein, a C18-HPLC column for $\mathrm{CNBr}$ cleavage, a sulfopropyl column to transfer the peptide from an acidic milieu to a neutral milieu and a second Ni-NTA column to remove the fusion protein. ${ }^{[8]}$ The sulfopropyl and the Ni-NTA columns were operated in a tandem configuration where the Ni-NTA acted more like a filter by allowing the peptide to pass quickly but retaining the fusion protein. This allowed the procedure to be accomplished within minutes.

Our fear that the amyloid peptide aggregates already during its preparation is real. We observed that the quality of the preparation decreased when the column and the tubing were used several times. If we flushed the entire tubing with a Congo red solution, all parts which were in contact with the amyloid peptide were stained.[9] This shows that aggregation indeed starts already as soon as it is transferred to physiological buffers. Congo red is the dye originally used to stain the amyloid plaques in thin sections from brains.

Funnily enough, the human amyloid peptide contains two neighboring histidines. But nevertheless, the peptide does not bind to nickel-NTA columns in $12 \mathrm{mM}$ Tris- $\mathrm{HCl} \mathrm{pH}$ 8. This may be an indication that the peptide adopts a secondary structure which brings the two histidines in a position which is not suitable for Ni-NTA binding.

\section{Too much Affinity Label}

When we designed different histidine-tags, we were concerned that we would make the binding to nickel too strong. As shown above, a small peptide is able to strip off the nickel ion from an IDA-column. Although the NTA-column binds the nickel ion much more strongly, Herbert Andres, a colleague from the diagnostic department, succeeded to construct an affinity tag which out-competed even this. A protein with the carboxy-terminal extension HHHHHHC transformed the light blue nickel-NTA column into a white column.

The intention of Herbert was to use the affinity tag not only for purification but also for covalent and site-directed immobilization. Hence the addition of a cysteine residue at the very end of the protein.

A single free sulfhydryl group is not able to strip the nickel from the NTA-column nor is it able to elute a protein tagged with six histidines. For instance, it is possible to use mercapto ethanol during the chromatographic procedure. But dithioerythritol (DTE) which has two sulfhydryl groups strips off the nickel from the column as does a label with one free sulfhydryl group embedded into a histidine-rich surrounding.

\section{Selective S-S Bridge Thanks to Reversible Immobilization}

Chemical synthesis by solid phase procedures provides rapid and systematic access to peptides. But solid-phase synthesis for peptides has its limitations: (i) if the length of the peptide exceeds 40-60 amino acids, (ii) if the peptide contains disulfide bridges, and (iii) if the peptide contains a stretch of hydrophobic amino acid residues.

Using similar fusion protein constructs as we used to express the amyloid peptide, we established a method for the recombi- 
nant production of disulfide bridge containing peptides with chain length of 35 to 50 residues.

Again, immobilization to a solid support to perform a chemical reaction was the key. Selective oxidation of disulfide bridge without concatamer formation was performed on a nickel-NTA column. The bulky repeat of 19 NANP units kept the proteins apart in order to allow exclusively intra-chain disulfide bridges. After application, the proteins were first reduced and then oxidized.

The second step, cleaving the fusion tail, was performed exactly as with the amyloid peptide. Since we had many different constructs at hand, we were able to get information of the cleavage efficiency of the cleavage-on-a-HPLC-column method. ${ }^{[10]}$

\section{Natural Proteins Retained on Nickel-NTA Columns}

Very soon we realized that most expression systems generate natural proteins which have histidines able to bind to Ni-NTA columns. A purification that does not take this into account can lead to confusing outcomes.

One of our projects of high priority had a fulminate start proceeding to a sudden end. This project was based on the observation that loss-of-function mutations of PCSK9 result in a low level of low-density lipoprotein (LDL). ${ }^{[11]}$ PCSK9 is a member of the serine protease family. It was tempting to initiate a project. Finding an inhibitor of that protease could be an elegant method to shift the bad cholesterol (LDL) level to a healthier value.

The gene sequence of PCSK9 was known and even the expression and purification was described. ${ }^{[12]}$ Only the natural substrate was not known, but the authors of that paper made some suggestions based on model peptide substrates. Our expression and purification protocol was almost identical to the described method consisting of a concanavalin A, a nickel-NTA and an anion exchange column to isolate the His-tagged protein. We were happy to find a virtually pure enzyme with proteolytic activity. To positively identify the protein, we used western blotting and a protease activity assay based on FRET-substrates.

During the scale-up of the purification procedure Nicole Soder analyzed the elution profile of the anion exchange column. She noticed that the peak protease activity and the peak western-blot signal were different by one fraction. Nicole repeated the experiment, but used a shallower gradient for elution and smaller fractions. Thereby it became evident that the protease activity did not arise from PCSK9 but by a contamination which turned out to be a cathepsin L-like enzyme. Cathepsin L shares many properties with PCSK9 which are relevant for its purification. Despite a huge collection of different protease substrates, we could not identify any proteolytic activity after removing all traces of cathepsin L in the PCSK9 preparation. Some of my colleagues were distrustful that I had not worked hard enough. Relief came when experts in the field[13] confirmed that PCSK9 performs only one cleavage in its lifetime, namely the self-cleavage of its prosegment. Afterward, PCSK9 serves functions other than proteolysis.

Take home message: It is a good idea to check if proteins copurify. We could have done better right at the beginning by passing a fermentation broth without PCSK9 over a concanavalin A, a Ni-NTA and an anion exchange column and check for protease activity.

\section{Licensing to Qiagen}

Roche is a pharmaceutical and diagnostic company and it was not intended to invest in technical methods unless it was required for our in-house projects. Therefore, the His-tag technology was licensed out to Qiagen and commercialized. But all examples described above took place in the laboratories of Roche Basel.

The polyhistidine affinity tail project emerged in a very research-friendly environment. In those days, researchers in Roche were encouraged by their management to spend some time apart from their core projects. But none of our team could envisage the broad applicability of that technique which goes far beyond protein purification.

\section{Acknowledgment}

I would like to thank all colleagues mentioned in the text or in the cited publications and in particular also all those who are not mentioned by name but provided important services in the background. I thank Dr. Jeremy Beauchamp for reviewing the manuscript to render it comprehensible also for non-protein insiders. Also, I thank F. Hoffmann - La Roche AG for offering me an innovation friendly research atmosphere which allowed me to become a witness of the rapid evolution of genomics and proteomics.

Received: February 18, 2020

[1] J. Porath, B. Olin, Biochem. 1983, 22, 162, DOI: 10.1021/bi00276a015.

[2] J. C. Smith, R. B. Derbyshire, E. Cook, L. Dunthorne, J. Viney J, S. J. Brewer, H. M. Sassenfeld, L. D. Bell. Gene, 1984, 32, 321, DOI: 10.1016/03781119(84)90007-6.

[3] H. Döbeli, E. Hochuli, Patent Appl. CH 2782/86, 1986.

[4] E. Hochuli, H. Döbeli, A. Schacher, J. Chromatogr. 1987, 411, 177, DOI: 10.1016/s0021-9673(00)93969-4.

[5] H. Döbeli, R. Gentz, W. Jucker, G. Garotta , D.W. Hartmann, E. Hochuli, J. Biotechnol. 1988, 7, 199.

[6] H. Döbeli, B. Eggimann, R. Gentz, E. Hochuli, D. Stüber, Europäische Patentanmeldung 88103740.2, 1988.

[7] E. Hochuli, W. Bannwarth, H. Döbeli, R. Gentz, D. Stüber, Nat. Biotechnol. 1988, $6,1321$.

[8] H. Döbeli, N. Draeger, G. Huber, P. Jakob, D. Schmidt, B. Seilheimer, D. Stüber, B. Wipf, M. Zulauf, Nat. Biotechnol. 1995, 13, 1142. DOI: 10.1038/ nbt0995-988.

[9] B. Seilheimer, B. Bohrmann, L. Bondolfi, F. Müller, D. Stüber, H. Döbeli, J. Struct. Biol. 1997, 119, 59, DOI: 10.1006/jsbi.1997.3859.

[10] H. Döbeli, H. Andres, N. Breyer, N. Draeger, D. Sizmann, M. T. Zuber, B. Weinert, B. Wipf, Protein Expression Purif. 1998, 12, 404, DOI:10.1006/ prep.1997.0844.

[11] R. Q. Do, R. A. Vogel, G. G. Schwartz, Curr. Cardiol. Rep. 2013, 15, 345, DOI: $10.1007 / \mathrm{s} 11886-012-0345-\mathrm{z}$.

[12] S. Naureckiene, L. Ma, K. Sreekumar, U. Purandare, C. F. Lo, Y. Huang, L. W. Chiang, J. M. Grenier, B. A. Ozenberger, J. S. Jacobsen, J. D. Kennedy, P. S. DiStefano, A. Wood, B. Bingham, Arch. Biochem. Biophys. 2003, 420, 55, DOI: 10.1016/j.abb.2003.09.011

[13] N. G. Seidah, Z. Awan, M. Chrétien, M. Mbiky, Circ. Res. 2014, 114, DOI: 10.1161/CIRCRESAHA.114.301621

\section{License and Terms}

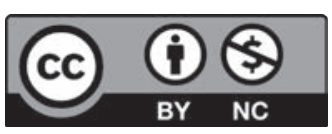

This is an Open Access article under the terms of the Creative Commons Attribution License CC BY_NC 4.0. The material may not be used for commercial purposes.

The license is subject to the CHIMIA terms and conditions: (http:// chimia.ch/component/sppagebuilder/?view=page \&id=12).

The definitive version of this article is the electronic one that can be found at doi:10.2533/chimia.2020.363 